\title{
Quantitative Mapping of Individual Voxels in the Peritumoral Region of Glioblastoma to Distinguish Between Tumor Infiltration and Edema
}

\author{
Archya Dasgupta \\ Sunnybrook Health Sciences Centre \\ Benjamin Geraghty \\ Sunnybrook Health Sciences Centre \\ Pejman Jabehdar Maralani \\ Sunnybrook Health Sciences Centre \\ Nauman Malik \\ Sunnybrook Health Sciences Centre \\ Michael Sandhu \\ Sunnybrook Health Sciences Centre \\ Jay Detsky \\ Sunnybrook Health Sciences Centre \\ Chia-Lin (Eric) Tseng \\ Sunnybrook Health Sciences Centre \\ Hany Soliman \\ Sunnybrook Health Sciences Centre \\ Sten Myrehaug \\ Sunnybrook Health Sciences Centre \\ Zain Husain \\ Sunnybrook Health Sciences Centre \\ James Perry \\ Sunnybrook Health Sciences Centre \\ Angus Lau \\ Sunnybrook Health Sciences Centre \\ Arjun Sahgal \\ Sunnybrook Health Sciences Centre \\ Gregory Czarnota ( $\nabla$ gregoryczamota.submissions@gmail.com ) \\ Sunnybrook Health Sciences Centre https://orcid.org/0000-0002-0519-2182
}

\section{Research Article}

Keywords: Radiomics, Magnetic resonance imaging (MRI), Glioblastoma multiforme (GBM), Peritumoral region, Edema

Posted Date: March 1st, 2021

DOI: https://doi.org/10.21203/rs.3.rs-263971/v1

License: (a) (i) This work is licensed under a Creative Commons Attribution 4.0 International License. Read Full License 


\section{Abstract}

Purpose: The peritumoral region (PTR) in glioblastoma (GBM) represents a combination of infiltrative tumor and vasogenic edema, which are indistinguishable on magnetic resonance imaging (MRI). We developed a radiomic signature by using imaging data from low grade glioma (LGG) (marker of tumor) and PTR of brain metastasis (BM) (marker of edema) and applied it on the GBM PTR to generate probabilistic maps.

Methods: 270 features were extracted from T1-weighted, T2-weighted, and apparent diffusion coefficient maps in over 3.5 million voxels of LGG (36 segments) and BM (45 segments) scanned in a 1.5 T MRI. A support vector machine classifier was used to develop the radiomics model from approximately $50 \%$ voxels (downsampled to $10 \%$ ) and validated with the remaining. The model was applied to over 575,000 voxels of the PTR of 10 patients with GBM to generate a quantitative map using Platt scaling (infiltrative tumor vs. edema).

Results: The radiomics model had an accuracy of 0.92 and 0.79 in the training and test set, respectively (LGG vs. BM). When extrapolated on the GBM PTR, 9 of 10 patients had a higher percentage of voxels with a tumor-like signature over radiological recurrence areas. In 7 of 10 patients, the areas under curves (AUC) were $>0.50$ confirming a positive correlation. Including all the voxels from the GBM patients, the infiltration signature had an AUC of 0.61 to predict recurrence.

Conclusion: A radiomic signature can demarcate areas of microscopic tumors from edema in the PTR of GBM, which correlates with areas of future recurrence.

\section{Introduction}

Radiomics involves advanced computational analysis of images linked to biological characteristics or clinical outcomes, which can be derived from different imaging modalities performed as a part of standard management protocols [1]. For patients with brain tumors, magnetic resonance imaging (MRI) serves an integral role in diagnosis, treatment planning, response assessment, and surveillance [2]. Radiomics using MRI has been widely applied in glioblastoma (GBM), showing reasonable effectiveness in characterizing molecular features (radiogenomics), clinical outcomes, tumor segmentation, and follow up assessment [3].

On MRI, several distinct compartments are identified in GBM, including the contrast-enhancing tumor core, central necrotic region, and the peritumoral region (PTR). The PTR is recognized as a non-enhancing area surrounding the tumor core identified as T2-FLAIR hyperintensity. Pathologically, the PTR harbors both microscopic tumor infiltration and vasogenic edema and is believed to play an essential role in disease progression [4-6]. The current imaging tools cannot differentiate infiltrative tumor versus edema in the PTR. Low grade glioma (LGG) and the PTR of patients with brain metastasis (BM) both appear to have similar radiologic appearances with the GBM PTR. However, in LGG, the region represents microscopic tumor cells, while in BM, the PTR constitutes vasogenic edema without infiltrative disease.

In this study, we used a radiomics approach to differentiate microscopic tumors from edema in individual voxels, with LGG and BM PTR serving as prototypes for the two groups. Subsequently, we have taken a novel approach of applying the radiomics model from the two tumor types to each voxel of the PTR of GBM on RT planning MRI to generate quantitative maps indicating tumor or edema on a probabilistic scale. Regions indicative of infiltrative tumors were finally correlated to the future areas of radiological disease recurrence.

\section{Materials And Methods}

\section{Patient Selection}

This retrospective study was approved by the ethics committee of the Sunnybrook Health Sciences Centre, Toronto, and the requirement for informed consent of patients was waived. Patients with BM and LGG treated between January 2014 and December 2018 were identified from an institutional database. To minimize interscanner variability, patients were only considered for inclusion if their imaging data were obtained using one of two identical scanners from a single vendor.

Patients with LGG were required to have a histological diagnosis of grade 2 glioma (astrocytoma or oligodendroglioma) from the index lesion without any pathological or radiological features suspicious for high grade glioma (HGG). Patients receiving prior chemotherapy or RT were excluded. Post-operative patients were included, with the surgical cavity and tracks were excluded from the segmented region. For patients with BM, we included treatment-naïve tumors with a known primary malignancy site, with unequivocal metastasis on MRI. Tumors with suspected hemorrhage or prior history of any RT to the brain or head-neck region were excluded.

The exploratory cohort included patients with GBM treated with adjuvant RT undergoing planning scans in the same MRI scanner (having all the three sequences: T1, T2, DWI). All the patients were required to have a well-defined radiological progression involving the PTR and good quality MRI scans available at the time of recurrence (at least T1c sequence).

\section{MRI Protocol, Image Preprocessing, Segmentation}

Imaging was performed on one of the two GE Signa HDxT 1.5T MRI scanners using an 8-channel head coil (General Electric Medical Systems, Waukesha, WI, USA). Three sequences were included in the current study: gadolinium (Gd) enhanced 3D T1-weighted FSPGR (T1c); Gd-enhanced T2 fluid-attenuated inversion-recovery PROPELLOR (T2f); and diffusion-weighted imaging-derived apparent diffusion coefficient maps (ADC). 
Data handling and scripting were performed in Matlab R2018b (The Mathworks, Inc., Natick, MA, USA). Following image registration, the T1c, T2f and ADC volumes were combined into single workspaces for each patient using the software platform ITK-SNAP (http://www.itksnap.org). Manual segmentation was performed initially by a radiation oncology fellow (AD), and all cases were individually reviewed by a neuroradiologist (PM, with 8 years of experience) and a neuro-radiation oncologist (AS, with 15 years of experience), and the segments were modified based on consensus amongst the three physicians. For BMs, the PTR region was segmented by identifying areas with T2-hyperintensity surrounding the enhancing tumor. The LGGs were segmented to include the tumor, excluding the surgical cavity for post-operative cases. For the exploratory GBM cohort, the PTR was manually drawn in the RT planning MRI (post-surgery), excluding the surgical cavity and residual enhancing lesion.

\section{Feature Extraction}

The feature extraction was performed using Pyradiomics software (default set of features) (http://www.pyradiomics.io/pyradiomics.html). Image discretization using a fixed gray level bin-width rather than a fixed number of bins was performed to improve reproducibility [7, 8].

The feature set included the following: 18 first-order statistical features; 21 gray level co-occurrence matrix (GLCM) features; 16 gray level size zone (GLSZM) features; 16 gray level run length matrix (GLRLM) features; 5 neighboring gray tone difference matrix (NGTDM) features, and 14 gray level dependence matrix (GLDM) features. A detailed description of the features can be found on the Pyradiomics website. This feature set was extracted from each of the T1c, T2f, and ADC scans for a total of 270 features per LGG and BM voxels. Feature extraction was performed for each voxel using a local patch of $5 \times 5 \times 5$ voxels using a sliding window technique.

\section{Classification}

Lesions (BM and LGG) were divided into training and testing as follows: the lesion list was shuffled, and entire lesions were successively included in the training cohort until the number of voxels totaled at least 1,000,000, and the remaining lesions were assigned to the testing cohort. To handle the large amount of data, the training set was linearly subsampled by taking the 270 features from every 100 th voxel across the lesions, resulting in a final training set of 20000 voxels, divided evenly between LGG and BM.

All standardization, model fitting, and assessment were performed using Scikit-Learn (https://scikit-learn.org/stable). The features in the training set were standardized by removing their means and scaling to unit variance. The testing set was scaled using the means and variances computed from the training set. The SVM classifier based on LIBSVM was used with a radial-basis-function kernel. The regularization parameter $\mathrm{C}$ was set to 1 , and the kernel coefficient gamma was set to 0.0037 , which is the reciprocal of the number of features in the training set. Probability estimates were calibrated during model fitting with internal 5-fold cross-validation using the built-in implementation of Platt scaling provided through LIBSVM.

\section{Mapping of Post-operative Glioblastoma}

As with the LGG and BM patients, T1c, T2f, and ADC scans obtained for the GBM were subjected to the same post-processing steps outlined above. The extracted features were standardized and subjected to the previously described SVM classifier, and probability estimates of LGG- or BM-like signatures were generated. The classification probability for each voxel was then placed into its corresponding location in the original peritumoral segments to generate 3D probability edema maps for each of the post-operative GBM cases.

Follow-up T1c scans corresponding to the presentation of radiological progression were obtained and then rigidly registered to the original post-operative T1c scans. After registration, regions of enhancing tumor progression were manually segmented using ITK-SNAP. Regions of progression that fell outside of the postoperative PTR were not considered for analysis. Areas of the original peritumoral edema that intersected with the progression regions were labeled as recurrence PTR and those that did not were labeled as non-recurrence PTR.

A detailed description of the study methodology has been included in Supplementary file 1.

\section{Results}

\section{Patient details}

We identified a total of 45 segments from 29 patients with BM and 36 segments of LGG from 34 patients. A total of 2166300 LGG and 1672488 BM voxels were extracted. For patients with BM, the most common primary site was lung. The LGG group included grade 2 astrocytoma ( $n=25$ ), grade 2 oligodendroglioma $(n=11)$. Pertinent features for the two groups across the training and test sets have been presented in Table 1.

For the 10 patients with GBM, the median age was 60 years (range 44 to 74 years). Eight patients were treated with adjuvant RT 60 Gy in 30 fractions, with one patient each receiving $34 \mathrm{~Gy}$ in 10 fractions and $40 \mathrm{~Gy}$ in 15 fractions. Except for the patient treated with $34 \mathrm{~Gy}$ in 10 fractions, all received concurrent temozolomide and adjuvant temozolomide (median 4 cycles, range 2 to 6 cycles until progression). The details of treatment, outcomes, and different indices for classifier performance and recurrence prediction have been summarized in Table 2.

\section{Classification Matrix}

For the classification of individual voxels, the training set consisted of 270 features from 20000 voxels. The sensitivity, specificity, and accuracy were 0.92 , 0.93 , and 0.92 , respectively. The test cohort consisted of 1103463 voxels (15 lesions) and 669462 voxels (24 lesions) from LGG and BM, respectively. The 
trained classifier was applied to each of the lesions assigned to the testing cohort separately, and the results were aggregated. The testing set consisted of 270 features from 1772925 voxels, and the sensitivity was 0.86 , the specificity was 0.68 , and the overall accuracy was 0.79 with an area under curve (AUC) value of 0.86 (Supplementary file 2). Since there was an unequal number of voxels across the LGG and BM classes, it is important to report the balanced accuracy ([sensitivity + specificity]/2), which was 0.77 .

\section{Outcomes in Glioblastoma Cohort}

A total of 576420 voxels were mapped using the developed radiomics model. The radiotherapy planning MRI scans with the plotted maps in the PTR, and co-registered MRI during recurrence has been shown in Figure 1 on a per-patient basis. The represented dominant areas of tumor recurrence have been marked with arrows, and visual inspection indicates a relation with tumor-like voxels (bright red) as derived from the radiomics model in the majority of the patients. A higher fraction (percentage of area $>0.5$ in recurrence-PTR overlap: percentage of area $>0.5$ in non-recurrence-PTR overlap) of tumor-like voxels was noted in 9 of 10 patients (except patient 1, who had multicentric disease). In other patients, the mean fraction was 1.78 , range 1.03 to 4.33 . The density normalized histograms obtained from the individual voxel values in the recurrence-PTR and non-recurrence-PTR for individual patients are shown in Figure 2. On general inspection, the red region (voxel with recurrence PTR) peaking towards the right corner (higher possibility of the tumor-like region) suggests positive relation. The AUC values (using a threshold of $>0.5$ on the probabilistic mapping scale) for tumor-like region and recurrence was $>0.50$ in 7 of 10 patients confirming positive correlation (except patient numbers 1, 6, and 10). For the other 7 patients with positive correlation, the mean AUC was 0.65 , range 0.58 to 0.69 . For all the voxels combined from all the 10 patients (including the outliers), the overall AUC was 0.61 . The combined histogram and AUC from all the voxels in 10 patients have been represented in Figure 3.

\section{Discussion}

The presence of an infiltrative tumor in the PTR region of GBM has been well established in GBM from several autopsy and surgical series [4-6]. Eidel et al. had demonstrated that although PTR's average cell density was comparable to the contrast-enhancing region, the PTR had a higher proportion of viable tumor cells (89\%) than the contrast-enhancing region (60\%) [6]. Following standard treatment with adjuvant chemoradiotherapy in patients with GBM, approximately $80-90 \%$ recurrences arise in the tumor margin and the PTR even in cases of complete resection of enhancing disease [9, 10]. There has been an increasing interest in understanding the role of the PTR in molecular pathogenesis, as the residual cells along the resection margin and in the surrounding region can represent resistant and rapidly proliferating clones [11]. The GBM-initiating cells or stem-like cells in the PTR have been associated with significant cellular plasticity and alternate molecular pathways, suggesting an aggressiveness as well as migratory potential, which can lead to disease recurrence $[12,13]$.

Although the microscopic tumor contents of the PTR is not appreciated with standard MRI sequences, several groups have attempted identifying areas of microscopic disease using sequences like DWI, dynamic susceptibility-weighted contrast-enhanced perfusion imaging (DSC), diffusion tensor imaging (DTI), magnetic resonance spectroscopy, and relaxometry $[5,14-16]$. While several studies have correlated the MRI findings with targeted tissue specimens with reasonable radiology-histology agreement, temporal correlation with specific areas of tumor progression has been lacking. Recently, studies have undertaken a quantitative or radiomics-based approach in mapping the PTR to generate probabilistic maps of tumor infiltration [17-20]. Akbari et al. used pre-operative T1, T1c, T2, T2f, DTI, and DSC sequences to extract radiomic features with the classifier trained, dividing the PTR's immediate and distant regions as prototypes for infiltration vs. no-infiltration [17]. Using the signature developed from the retrospective cohort, they had demonstrated an accuracy of $91 \%$ in associating the recurrence regions in the replication cohort. Rathore et al. had further expanded the methodology by including more imaging parameters like texture features and tested the model on a set of 59 patients showing an accuracy of $90 \%$ [18]. Consideration is to be given to the fact that the model was trained based on distance from the tumor core, and most of the recurrences are known to occur along the cavity in the proximal aspect of PTR, which can influence the classifier performances. In another study involving 82 tissue biopsies from 18 patients with GBM, Hu et al. had developed a radiomics model to differentiate high-infiltration vs. low-infiltration of tumors (cut-off of $80 \%$ tumor nuclei) in the PTR [19]. They had achieved an accuracy of $82 \%$ in the validation cohort. One significant difference compared to our study had been the difference in endpoint (tumor vs edema). With the histopathological correlation being a significant contribution of the study, the manual placement of segments corresponding to the biopsy site $(8 \times 8 \times 8$ voxels) might include neighbouring voxels with different tumor content. In a recent report by Yan et al. a neural network classifier was used to develop a radiomics model to map the PTR of pre-operative MRI, trained on extracting features from recurrence vs no-recurrence [20]. An accuracy of $78 \%$ was achieved on a validation group of 20 patients with GBM. One of the challenges in the some of the studies mentioned above lie in correlating the pre-operative imaging and registration with post-operative scans where the anatomy can be significantly altered following surgery and radiotherapy [21].

Our study undertook a novel approach to use a different group of tumors to serve as the prototype for tumor and edema. The post-operative scans were registered to MRIs at the time of recurrence (as opposed to the pre-surgical scans), which is likely to preserve a better spatial relation of individual segments to study selected regions showing disease progression. The study presented here supports the hypothesis of radiomics-based demarcation of the structurally distinct cellular regions (infiltrative tumor) from vasogenic edema affecting the extracellular compartment. Furthermore, in 7 of 10 patients, a relation was established between areas indicative of infiltrative tumor and subsequent recurrences, strengthening the study results. Analyzing the possible reasons for poor performance for the three patients: patient 1 presented with multicentric GBM, while patient 6 had progression along the edge of the cavity along the dura. No discernible reason could be identified for patient 10. It is important to note that the recurrence likely started over small regions that further progressed (when MRI was obtained) to involve surrounding areas with lesser or no tumor infiltration at the radiation planning stage, possibly undermining the AUC values. 
A possible implication for pre-operative mapping of the PTR can be in surgical resection of regions with a likely higher burden of microscopic disease. Few studies had shown a potentially positive impact on survival in patients undergoing supratotal resection or "FLAIRectomy" [22, 23]. Another potential application for the current study will be in guiding RT target volumes, specifically targeting areas of infiltrative tumor, as the standard practice includes a wider margin in an empirical manner [24]. Identifying and excluding areas of reactive edema with a lower likelihood of microscopic disease from the target volumes can help reduce treatment toxicities and adopt individualized target volumes. In a phase 2 randomized trial, Kumar et al. had shown that larger radiation target volumes are associated with worse quality of life [25]. A recently conducted phase 1/2 trial by Azoulay et al. using a $5 \mathrm{~mm}$ clinical target volume (CTV) beyond the gross tumor volume to deliver 5- fraction stereotactic radiosurgery along with concurrent temozolomide has been shown to be a safe strategy in newly diagnosed GBM [26]. However, the authors had empirically included an isotropic margin of $5 \mathrm{~mm}$ (limiting at the anatomical borders like calvarium, falx, tentorium) without any attempt to include the PTR. The work presented here will be valuable to enable safe margin reduction. Our center is planning a clinical trial investigating MRI guided linear accelerator (MR-LINAC) treatment with 60 Gy in 30 fractions with a 5 mm CTV that would include T2f indicative of microscopic tumor using this method and treatment plan adaption.

Future steps will include the standardization of imaging features from different MRI scanners to test the robustness and extend the clinical utility. Although only 10 patients with GBM were included in the exploratory cohort, the inclusion of over half a million voxels tested the stability of the radiomics model. Future studies will be undertaken to expand the GBM cohort and also study the progression dynamics on subsequent MRIs following the first radiological progression. Also, a higher fraction of microscopic disease might be one of the attributing factors of progression in that particular location, with an expected complex interaction of tumor hypoxia and nests of resistant clones. Integration of the developed signature (tumor vs. edema) with other radiomic signatures (extracted from treatment-naïve co-registered recurrence segments) may help refine the model's performance.

\section{Conclusions}

A radiomics model developed using texture features from $T 1 \mathrm{c}, \mathrm{T} 2 \mathrm{f}$, and $\mathrm{ADC}$ MRI sequences can effectively differentiate infiltrative tumor from vasogenic edema in the PTR of GBM. Extrapolation of the radiomics model to individual voxels in the RT planning MRI for patients with GBM can be used to identify the regions with a higher likelihood of microscopic disease with a higher risk of disease recurrence.

\section{Declarations}

Acknowledgments: We would like to thank the patients and their caregivers involved in the study. Our sincere gratitude to the Terry Fox Foundation Program Project Grant from the Hecht Foundation for the funding support associated with the study.

Funding: Terry Fox Foundation Program Project Grant from the Hecht Foundation (1083) awarded to Gregory J. Czarnota. The funding bodies had no influence on the study design, data collection, analysis, interpretation of data, or the manuscript's writing.

Conflicts of interest:

Archya Dasgupta: None

Benjamin Geraghty: None

Pejman Maralani: None

Nauman Malik: None

Michael Sandhu: None

Jay Detsky: None

Chia-Lin Tseng: Travel accommodations/expenses \& honoraria for past educational seminars by Elekta, belongs to the Elekta MR-Linac Research Consortium, and advisor/consultant with Sanofi

Hany Soliman: None

Sten Myrehaug: Travel accommodations/expenses from Elekta AB. Research support from Novartis/AAA.

Zain Husain: Travel accommodations/expenses from Elekta.

James Perry: None

Angus Lau: None.

Arjun Sahgal: Advisor/consultant with AbbVie, Merck, Roche, Varian (Medical Advisory Group), Elekta (Gamma Knife Icon), BrainLAB, and VieCure (Medical Advisory Board)

Board Member: International Stereotactic Radiosurgery Society (ISRS)

Page 5/11 
Past educational seminars with Elekta AB, Accuray Inc., Varian (CNS Teaching Faculty), BrainLAB, Medtronic Kyphon

Research grant with Elekta $A B$

Travel accommodations/expenses by Elekta, Varian, BrainLAB

Elekta MR Linac Research Consortium, Elekta Spine, Oligometastases and Linac Based SRS Consortia

Gregory J. Czarnota: Funding received from the Terry Fox Foundation Program Project Grant.

Availability of data and material: Data will be made available on request to the corresponding author following institutional ethics committee protocols.

Code availability: The radiomic feature extraction was performed using freely available Pyradiomics software (default set of features)

(http://www.pyradiomics.io/pyradiomics.html). All standardization, model fitting, and assessment were performed using Scikit-Learn (https://scikit-

learn.org/stable). The SVM classifier was used based on LIBSVM with a radial-basis-function kernel.

\section{Author contributions:}

Conceptualization: AD, BG, AS, GJC; Methodology: All authors; Formal Analysis and investigation: All authors; Writing-original draft preparation: AD, BG, AS, GJC; Writing-review and editing: All authors; Project administration and supervision: AS, GJC; Funding acquisition: GJC

All the authors are in agreement and accountable for all the aspects of the work.

Ethics approval: The study was approved by the Research Ethics Board of Sunnybrook Health Sciences Centre (protocol number: 034-2020).

Consent to participate: Consent was waived for the retrospective study.

Consent for publication: Not applicable (anonymized data, imaging study).

Presentation: The initial results of the study were presented at the $62^{\text {nd }}$ annual meeting of the American Society for Radiation Oncology (ASTRO) in October 2020.

\section{References}

1. Lambin P, Rios-Velazquez E, Leijenaar R, et al (2012) Radiomics: Extracting more information from medical images using advanced feature analysis. Eur J Cancer 48:441-446. https://doi.org/10.1016/j.ejca.2011.11.036

2. Villanueva-Meyer JE, Mabray MC, Cha S (2017) Current Clinical Brain Tumor Imaging. Neurosurgery 81:397-415. https://doi.org/10.1093/neuros/nyx103

3. Chaddad A, Kucharczyk MJ, Daniel P, et al (2019) Radiomics in Glioblastoma: Current Status and Challenges Facing Clinical Implementation. Front Oncol 9:. https://doi.org/10.3389/fonc.2019.00374

4. Yamahara T, Numa Y, Oishi T, et al (2010) Morphological and flow cytometric analysis of cell infiltration in glioblastoma: a comparison of autopsy brain and neuroimaging. Brain Tumor Pathol 27:81-87. https://doi.org/10.1007/s10014-010-0275-7

5. Barajas RF, Phillips JJ, Parvataneni R, et al (2012) Regional variation in histopathologic features of tumor specimens from treatment-naive glioblastoma correlates with anatomic and physiologic MR Imaging. Neuro Oncol 14:942-954. https://doi.org/10.1093/neuonc/nos128

6. Eidel O, Burth S, Neumann J-O, et al (2017) Tumor Infiltration in Enhancing and Non-Enhancing Parts of Glioblastoma: A Correlation with Histopathology. PLoS One 12:. https://doi.org/10.1371/journal.pone.0169292

7. Duron L, Balvay D, Perre SV, et al (2019) Gray-level discretization impacts reproducible MRI radiomics texture features. PLoS ONE 14:. https://doi.org/10.1371/journal.pone.0213459

8. Carré A, Klausner G, Edjlali M, et al (2020) Standardization of brain MR images across machines and protocols: bridging the gap for MRI-based radiomics. Sci Rep 10:. https://doi.org/10.1038/s41598-020-69298-z

9. McDonald MW, Shu H-KG, Curran WJ, Crocker IR (2011) Pattern of failure after limited margin radiotherapy and temozolomide for glioblastoma. Int J Radiat Oncol Biol Phys 79:130-136. https://doi.org/10.1016/j.jirobp.2009.10.048

10. Petrecca K, Guiot M-C, Panet-Raymond V, Souhami L (2013) Failure pattern following complete resection plus radiotherapy and temozolomide is at the resection margin in patients with glioblastoma. J Neurooncol 111:19-23. https://doi.org/10.1007/s11060-012-0983-4

11. Lemée J-M, Clavreul A, Menei P (2015) Intratumoral heterogeneity in glioblastoma: don't forget the peritumoral brain zone. Neuro Oncol 17:13221332. https://doi.org/10.1093/neuonc/nov119

12. Glas M, Rath BH, Simon M, et al (2010) Residual Tumor Cells Are Unique Cellular Targets in Glioblastoma. Ann Neurol 68:264-269. https://doi.org/10.1002/ana.22036

13. Ruiz-Ontañon P, Orgaz JL, Aldaz B, et al (2013) Cellular plasticity confers migratory and invasive advantages to a population of glioblastomainitiating cells that infiltrate peritumoral tissue. Stem Cells 31:1075-1085. https://doi.org/10.1002/stem.1349 
14. Price SJ, Jena R, Burnet NG, et al (2006) Improved delineation of glioma margins and regions of infiltration with the use of diffusion tensor imaging: an image-guided biopsy study. AJNR Am J Neuroradiol 27:1969-1974

15. Yan J-L, Li C, Boonzaier NR, et al (2019) Multimodal MRI characteristics of the glioblastoma infiltration beyond contrast enhancement. Ther Adv Neurol Disord 12:1756286419844664. https://doi.org/10.1177/1756286419844664

16. Blystad I, Warntjes JBM, Smedby Ö, et al (2020) Quantitative MRI using relaxometry in malignant gliomas detects contrast enhancement in peritumoral oedema. Sci Rep 10:17986. https://doi.org/10.1038/s41598-020-75105-6

17. Akbari H, Macyszyn L, Da X, et al (2016) Imaging Surrogates of Infiltration Obtained Via Multiparametric Imaging Pattern Analysis Predict Subsequent Location of Recurrence of Glioblastoma. Neurosurgery 78:572-580. https://doi.org/10.1227/NEU.0000000000001202

18. Rathore S, M.d HA, Doshi J, et al (2018) Radiomic signature of infiltration in peritumoral edema predicts subsequent recurrence in glioblastoma: implications for personalized radiotherapy planning. JMI 5:021219. https://doi.org/10.1117/1.JMI.5.2.021219

19. Hu LS, Ning S, Eschbacher JM, et al (2015) Multi-Parametric MRI and Texture Analysis to Visualize Spatial Histologic Heterogeneity and Tumor Extent in Glioblastoma. PLoS One 10:e0141506. https://doi.org/10.1371/journal.pone.0141506

20. Yan J-L, Li C, van der Hoorn A, et al (2020) A Neural Network Approach to Identify the Peritumoral Invasive Areas in Glioblastoma Patients by Using MR Radiomics. Scientific Reports 10:9748. https://doi.org/10.1038/s41598-020-66691-6

21. Stewart J, Sahgal A, Lee Y, et al (2020) Quantitating Inter-fraction Target Dynamics during Concurrent Chemoradiation for Glioblastoma: A Prospective Serial Imaging Study. International Journal of Radiation Oncology, Biology, Physics 0: https://doi.org/10.1016/j.ijrobp.2020.10.002

22. Jackson C, Choi J, Khalafallah AM, et al (2020) A systematic review and meta-analysis of supratotal versus gross total resection for glioblastoma. J Neurooncol 148:419-431. https://doi.org/10.1007/s11060-020-03556-y

23. Certo F, Altieri R, Maione M, et al (2020) FLAIRectomy in Supramarginal Resection of Glioblastoma Correlates With Clinical Outcome and Survival Analysis: A Prospective, Single Institution, Case Series. Oper Neurosurg (Hagerstown). https://doi.org/10.1093/ons/opaa293

24. Tseng C-L, Stewart J, Whitfield G, et al (2020) Glioma consensus contouring recommendations from a MR-Linac International Consortium Research Group and evaluation of a CT-MRI and MRI-only workflow. J Neurooncol. https://doi.org/10.1007/s11060-020-03605-6

25. Kumar N, Kumar R, Sharma SC, et al (2020) Impact of volume of irradiation on survival and quality of life in glioblastoma: a prospective, phase 2, randomized comparison of RTOG and MDACC protocols. Neurooncol Pract 7:86-93. https://doi.org/10.1093/nop/npz024

26. Azoulay M, Chang SD, Gibbs IC, et al (2020) A phase I/II trial of 5-fraction stereotactic radiosurgery with 5-mm margins with concurrent temozolomide in newly diagnosed glioblastoma: primary outcomes. Neuro Oncol 22:1182-1189. https://doi.org/10.1093/neuonc/noaa019

\section{Tables}

Table 1: Patient and disease-related characteristics for low grade glioma and the brain metastasis 


\begin{tabular}{|c|c|c|}
\hline \multicolumn{3}{|l|}{ Brain metastasis } \\
\hline Features & $\begin{array}{l}\text { Training set } \\
(n=21)\end{array}$ & $\begin{array}{l}\text { Test set } \\
(n=24)\end{array}$ \\
\hline Number of patients & 14 & 15 \\
\hline \multicolumn{3}{|l|}{ Age } \\
\hline Median (Range) & $60(46-79)$ years & $63(46-76)$ years \\
\hline \multicolumn{3}{|l|}{ Gender } \\
\hline Male & 17 & 10 \\
\hline Female & 4 & 14 \\
\hline \multicolumn{3}{|l|}{ Primary } \\
\hline Non-small-cell Lung cancer & 6 & 11 \\
\hline Sarcoma & 5 & 3 \\
\hline Colorectal & 3 & 0 \\
\hline Renal cell carcinoma & 2 & 5 \\
\hline Others & 5 & 5 \\
\hline \multicolumn{3}{|l|}{ Volume } \\
\hline Median (Range) & $18.5(0.3-253.6) \mathrm{cc}$ & $19.9(0.7-154.3))$ \\
\hline Total number of pixels & 1003026 (downsampled) & 669462 \\
\hline \multicolumn{3}{|l|}{ Low grade glioma } \\
\hline Features & $\begin{array}{l}\text { Training set } \\
(n=21)\end{array}$ & $\begin{array}{l}\text { Test set } \\
(n=15)\end{array}$ \\
\hline Number of patients & 20 & 14 \\
\hline \multicolumn{3}{|l|}{ Age } \\
\hline Median (Range) & 43 (24-71) years & $39(22-68)$ years \\
\hline \multicolumn{3}{|l|}{ Gender } \\
\hline Male & 13 & 11 \\
\hline Female & 8 & 4 \\
\hline \multicolumn{3}{|l|}{ Histology } \\
\hline Astrocytoma & 14 & 11 \\
\hline Oligodendroglioma & 7 & 4 \\
\hline \multicolumn{3}{|l|}{ IDH status } \\
\hline Mutation & 18 & 9 \\
\hline No mutation & 3 & 6 \\
\hline \multicolumn{3}{|l|}{$1 p / 19 q$ status } \\
\hline Codeleted & 6 & 4 \\
\hline No codeletion & 12 & 9 \\
\hline Unknown & 3 & 2 \\
\hline \multicolumn{3}{|l|}{ Volume } \\
\hline Median (Range) & $36.6(4.5-149.6) \mathrm{cc}$ & $64.2(1.3-184.4) \mathrm{cc}$ \\
\hline
\end{tabular}

Page 8/11 
Table 2: Patient characteristics and outcomes for patients with glioblastoma

\begin{tabular}{|c|c|c|c|c|c|c|c|c|c|c|c|c|c|}
\hline $\begin{array}{l}\mathrm{Pt} \\
\text { no }\end{array}$ & $\begin{array}{l}\text { Age/ } \\
\text { Gender }\end{array}$ & Treatment & $\begin{array}{l}\text { Time to } \\
\text { the first } \\
\text { recurrence }\end{array}$ & $\begin{array}{l}\text { Final } \\
\text { Status }\end{array}$ & $\begin{array}{l}\text { Edema } \\
\text { total } \\
\text { volume }\end{array}$ & $\begin{array}{l}\text { Rec- } \\
\text { overlap } \\
\text { volume } \\
\text { (\% of } \\
\text { edema) }\end{array}$ & $\begin{array}{l}\text { Rec } \\
<0.5 \\
\text { Volume } \\
(\%)\end{array}$ & $\begin{array}{l}\text { Rec > } \\
0.5 \\
\text { Volume } \\
(\%)\end{array}$ & $\begin{array}{l}\text { No-rec } \\
<0.5 \\
\text { Volume } \\
(\%)\end{array}$ & $\begin{array}{l}\text { No-rec } \\
>0.5 \\
\text { Volume } \\
(\%)\end{array}$ & $\begin{array}{l}\text { Ratio of } \\
\text { percentage } \\
\text { Rec }>0.5 / \\
\text { No-rec } \\
>0.5\end{array}$ & $\begin{array}{l}\text { p- } \\
\text { value }\end{array}$ & AUC \\
\hline 1 & $74 / \mathrm{M}$ & $\begin{array}{l}34 \text { Gy/10 } \\
\text { No TMZ }\end{array}$ & 5 months & $\begin{array}{l}\text { Death } \\
7 \\
\text { months }\end{array}$ & $\begin{array}{l}32.95 \\
\mathrm{cc}\end{array}$ & $19 \%$ & $\begin{array}{l}0.89 \mathrm{cc} \\
(14 \%)\end{array}$ & $\begin{array}{l}5.28 \text { cc } \\
(85 \%)\end{array}$ & $\begin{array}{l}3.7 \text { cc } \\
(14 \%)\end{array}$ & $\begin{array}{l}22.86 \\
\mathrm{CC} \\
(85 \%)\end{array}$ & 1.00 & 0.314 & 0.45 \\
\hline 2 & $44 / F$ & $\begin{array}{l}60 \mathrm{~Gy} / 30 \\
\text { conc }+6 \\
\text { cycles } \\
\text { TMZ }\end{array}$ & $\begin{array}{l}10 \\
\text { months }\end{array}$ & $\begin{array}{l}\text { Death } \\
22 \\
\text { months }\end{array}$ & $\begin{array}{l}16.11 \\
\mathrm{cc}\end{array}$ & $60 \%$ & $\begin{array}{l}0.19 \mathrm{cc} \\
(2 \%)\end{array}$ & $\begin{array}{c}9.44 \mathrm{cc} \\
(98 \%)\end{array}$ & $\begin{array}{l}0.33 \mathrm{cc} \\
(5 \%)\end{array}$ & $\begin{array}{l}6.12 \mathrm{cc} \\
(95 \%)\end{array}$ & 1.03 & $<0.001$ & 0.58 \\
\hline 3 & $72 / \mathrm{M}$ & $\begin{array}{l}40 \mathrm{~Gy} / 15 \\
\text { conc+3 } \\
\text { cycles } \\
\text { TMZ }\end{array}$ & 8 months & $\begin{array}{l}\text { Death } \\
20 \\
\text { months }\end{array}$ & $\begin{array}{l}41.17 \\
\mathrm{CC}\end{array}$ & $36 \%$ & $\begin{array}{l}12.75 \\
\mathrm{cc} \\
(87 \%)\end{array}$ & $\begin{array}{l}1.87 \text { cc } \\
(13 \%)\end{array}$ & $\begin{array}{l}25.58 \\
\mathrm{cC} \\
(97 \%)\end{array}$ & $\begin{array}{l}0.82 \text { cc } \\
(3 \%)\end{array}$ & 4.33 & $<0.001$ & 0.67 \\
\hline 4 & $59 / \mathrm{M}$ & $\begin{array}{l}60 \mathrm{~Gy} / 30 \\
\text { conc+4 } \\
\text { cycles } \\
\text { TMZ }\end{array}$ & 8 months & $\begin{array}{l}\text { Death } \\
13 \\
\text { months }\end{array}$ & $\begin{array}{l}62.25 \\
\mathrm{cC}\end{array}$ & $22 \%$ & $\begin{array}{l}4.81 \mathrm{cc} \\
(35 \%)\end{array}$ & $\begin{array}{l}8.97 \text { cc } \\
(65 \%)\end{array}$ & $\begin{array}{l}31.26 \\
\mathrm{cc} \\
(65 \%)\end{array}$ & $\begin{array}{l}16.7 \text { cc } \\
(35 \%)\end{array}$ & 1.86 & $<0.001$ & 0.69 \\
\hline 5 & $56 / M$ & $\begin{array}{l}60 \mathrm{~Gy} / 30 \\
\text { conc+5 } \\
\text { cycles } \\
\text { TMZ }\end{array}$ & 8 months & $\begin{array}{l}\text { Alive } \\
15 \\
\text { months }\end{array}$ & $\begin{array}{l}20.05 \\
\mathrm{CC}\end{array}$ & $7 \%$ & $\begin{array}{l}0.19 \mathrm{cc} \\
(14 \%)\end{array}$ & $\begin{array}{l}1.16 \text { cc } \\
(85 \%)\end{array}$ & $\begin{array}{l}6.19 \text { cc } \\
(33 \%)\end{array}$ & $\begin{array}{l}12.35 \\
\mathrm{cc} \\
(66 \%)\end{array}$ & 1.29 & $<0.001$ & 0.67 \\
\hline 6 & $62 / M$ & $\begin{array}{l}60 \mathrm{~Gy} / 30 \\
\text { conc+5 } \\
\text { cycles } \\
\mathrm{TMZ}\end{array}$ & 9 months & $\begin{array}{l}\text { Death } \\
17 \\
\text { months }\end{array}$ & $\begin{array}{l}29.82 \\
\text { CC }\end{array}$ & $27 \%$ & $\begin{array}{l}4.77 \mathrm{cc} \\
(60 \%)\end{array}$ & $\begin{array}{l}3.13 \mathrm{cc} \\
(39 \%)\end{array}$ & $\begin{array}{l}14.26 \\
\mathrm{cc} \\
(65 \%)\end{array}$ & $\begin{array}{c}7.39 \text { cc } \\
(34 \%)\end{array}$ & 1.15 & $<0.001$ & 0.50 \\
\hline 7 & $49 / M$ & $\begin{array}{l}60 \mathrm{~Gy} / 30 \\
\text { conc+2 } \\
\text { cycles } \\
\mathrm{TMZ}\end{array}$ & 6 months & $\begin{array}{l}\text { Death } \\
10 \\
\text { months }\end{array}$ & $\begin{array}{l}139.09 \\
\text { CC }\end{array}$ & $15 \%$ & $\begin{array}{l}10.7 \mathrm{cc} \\
(52 \%)\end{array}$ & $\begin{array}{l}9.84 \mathrm{cc} \\
(48 \%)\end{array}$ & $\begin{array}{l}86.4 \mathrm{cc} \\
(73 \%)\end{array}$ & $\begin{array}{l}31.43 \\
\mathrm{cc} \\
(27 \%)\end{array}$ & 1.78 & $<0.001$ & 0.63 \\
\hline 8 & $61 / M$ & $\begin{array}{l}60 \mathrm{~Gy} / 30 \\
\text { conc+2 } \\
\text { cycles } \\
\text { TMZ }\end{array}$ & 5 months & $\begin{array}{l}\text { Death } \\
13 \\
\text { months }\end{array}$ & $\begin{array}{l}76.78 \\
\mathrm{cC}\end{array}$ & $40 \%$ & $\begin{array}{l}15.50 \\
(50 \%)\end{array}$ & $\begin{array}{l}15.22 \\
\mathrm{cC} \\
(49 \%)\end{array}$ & $\begin{array}{l}35.76 \\
\mathrm{cc} \\
(78 \%)\end{array}$ & $\begin{array}{l}9.84 \text { cc } \\
(21 \%)\end{array}$ & 2.33 & $<0.001$ & 0.68 \\
\hline 9 & $55 / M$ & $\begin{array}{l}60 \mathrm{~Gy} / 30 \\
\text { conc+5 } \\
\text { cycles } \\
\text { TMZ }\end{array}$ & $\begin{array}{l}10 \\
\text { months }\end{array}$ & $\begin{array}{l}\text { Death } \\
22 \\
\text { months }\end{array}$ & $\begin{array}{l}21.77 \\
\mathrm{cc}\end{array}$ & $8 \%$ & $\begin{array}{l}0.05 \mathrm{cc} \\
(3 \%)\end{array}$ & $\begin{array}{c}1.61 \mathrm{cc} \\
(97 \%)\end{array}$ & $\begin{array}{l}2.7 \text { cc } \\
(13 \%)\end{array}$ & $\begin{array}{l}14.26 \\
\mathrm{cC} \\
(86 \%)\end{array}$ & 1.13 & $<0.001$ & 0.64 \\
\hline 10 & $68 / M$ & $\begin{array}{l}60 \mathrm{~Gy} / 30 \\
\text { conc+2 } \\
\text { cycles } \\
\text { TMZ }\end{array}$ & 5 months & $\begin{array}{l}\text { Death } \\
7 \\
\text { months }\end{array}$ & $\begin{array}{l}120.34 \\
\mathrm{CC}\end{array}$ & $12 \%$ & $\begin{array}{l}12.43 \\
\mathrm{cc} \\
(87 \%)\end{array}$ & $\begin{array}{l}1.86 \text { cc } \\
(13 \%)\end{array}$ & $\begin{array}{l}93.17 \\
\mathrm{CC} \\
(88 \%)\end{array}$ & $\begin{array}{l}12.4 \mathrm{cc} \\
(12 \%)\end{array}$ & 1.08 & $<0.001$ & 0.49 \\
\hline
\end{tabular}

\section{Figures}



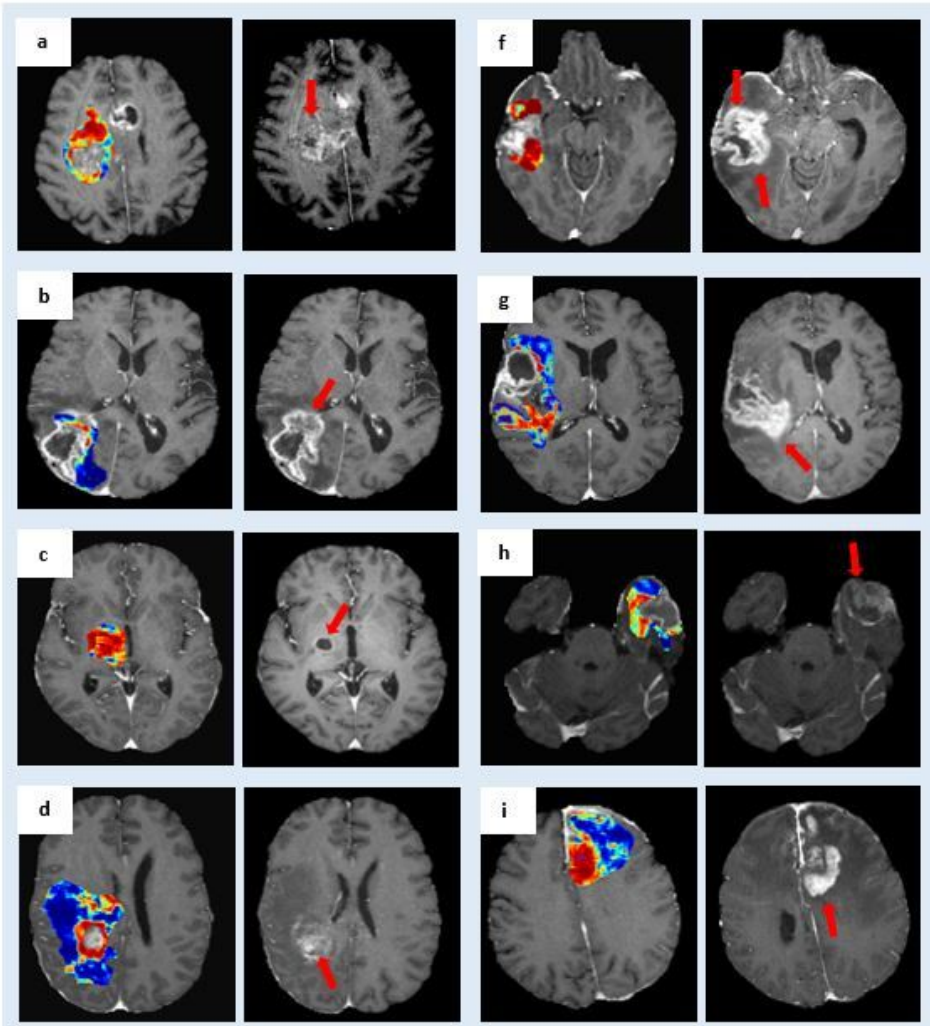

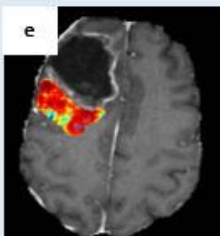

RT Planning

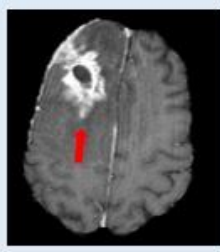

Recurrence

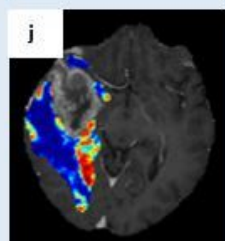

RT Planning

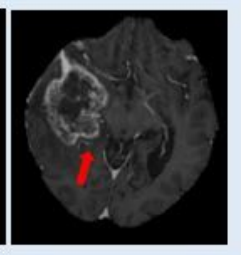

Recurrence

Figure 1

denotes radiation planning MRI with the probabilistic maps (left) and co-registered MRI obtained during disease recurrence (right) for patients with glioblastoma 1-10 (a-j). The bright red denotes the voxels with a higher probability of infiltrative tumor (low grade like signature), while bright blue represents areas with a higher likelihood of edema (brain metastasis like signature). The dominant areas of disease recurrence have been marked with red arrows
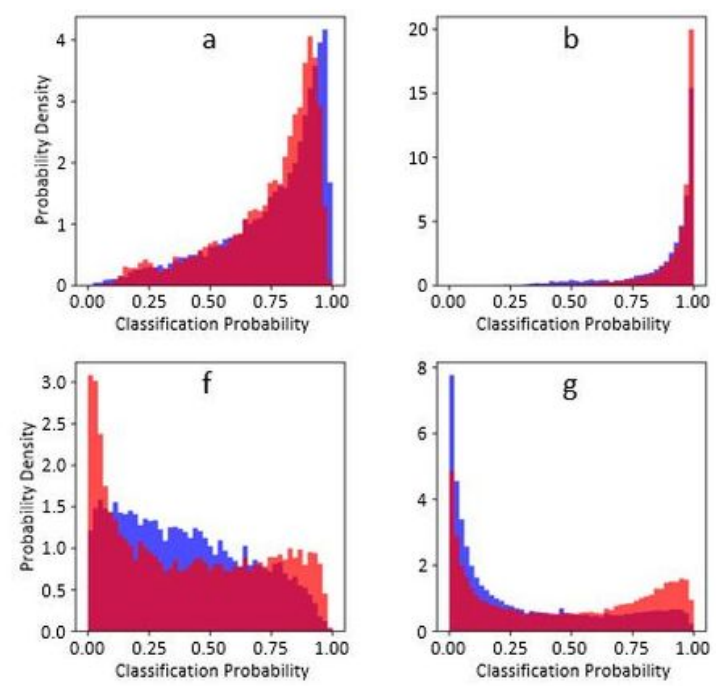
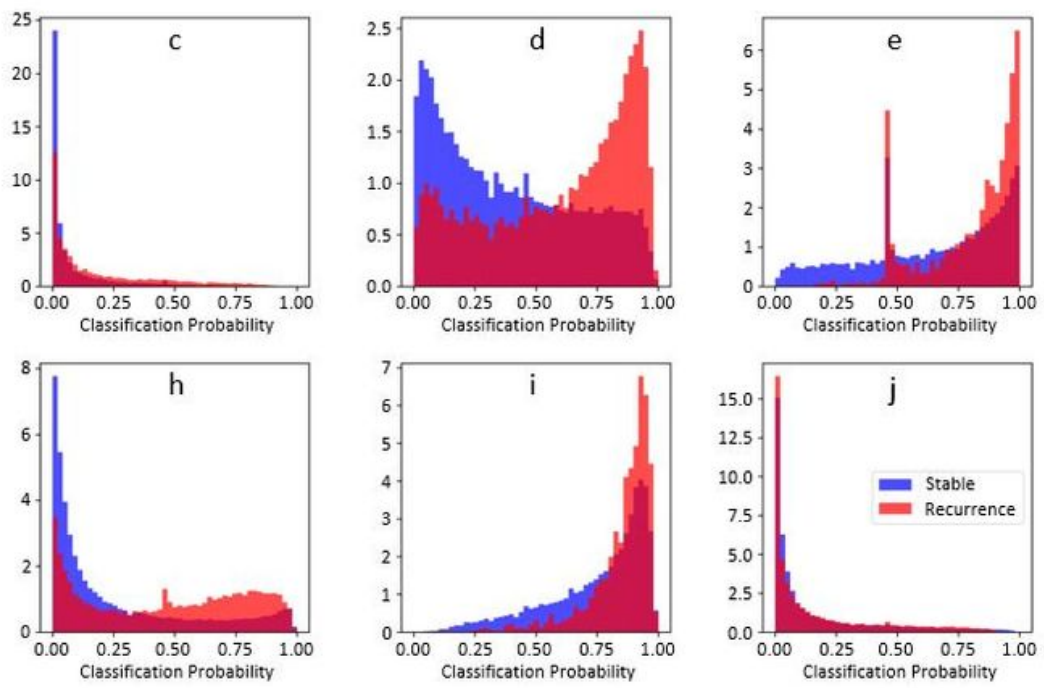
Figure 2

shows density-normalized histograms from all voxels from individual patients showing the distribution of voxels overlying recurrence-PTR (red) vs. nonrecurrence PTR (blue) for patients with glioblastoma 1-10 (a-j). The x-axis represents a continuous scale of 0 to 1 , with 0 showing the strongest likelihood of edema and 1 suggesting the most substantial tumor probability

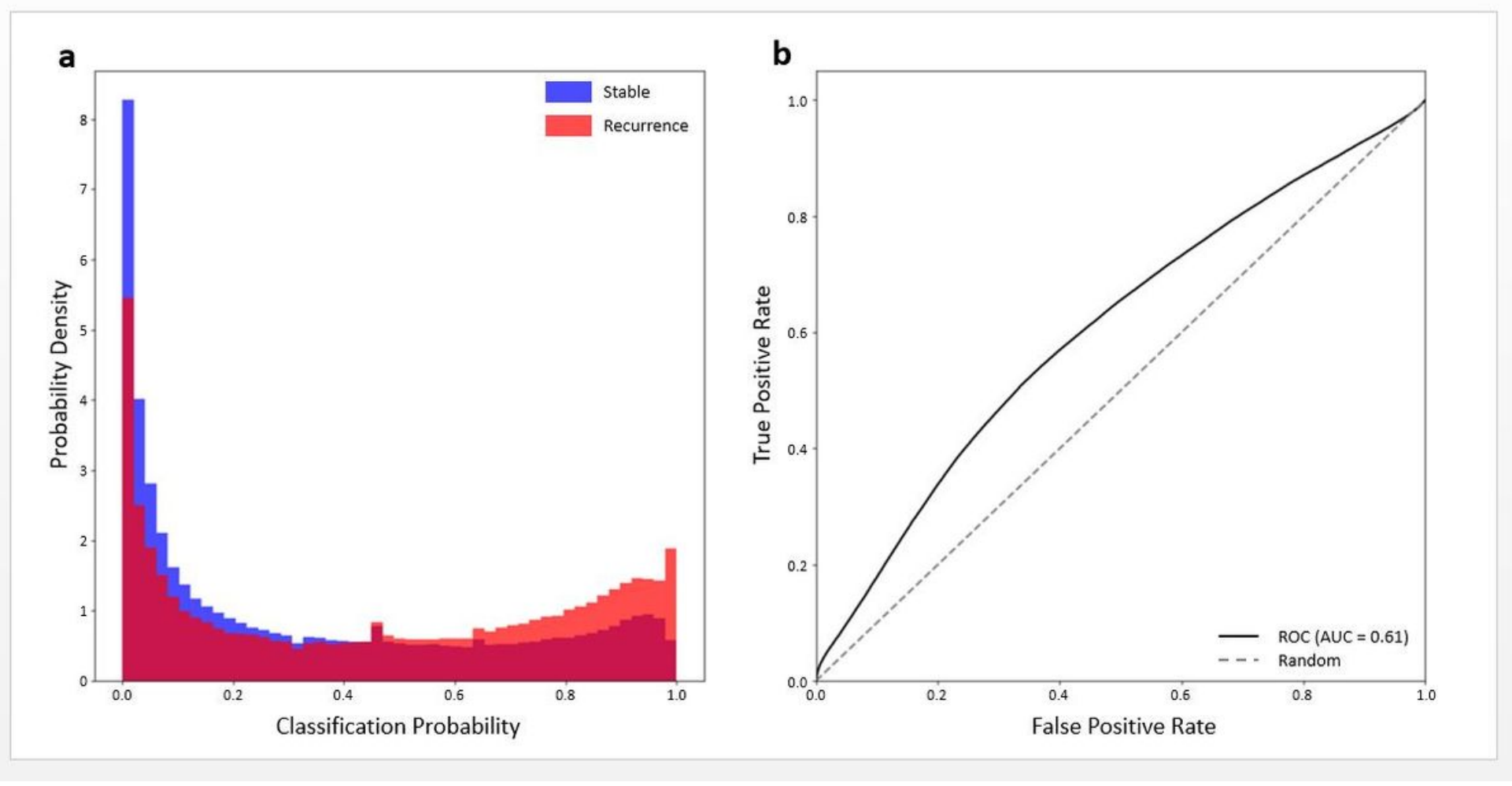

Figure 3

represents the combined density-normalized histogram obtained from all the voxels combined in 10 patients with glioblastoma (Fig. 3a) and the receiver operating characteristics curve resulting from all the voxels combined using a threshold of 0.5 (Fig. 3b)

\section{Supplementary Files}

This is a list of supplementary files associated with this preprint. Click to download.

- Supplementaryfile1textmethods.docx

- Supplementaryfile2Figure1ROCplottestsetLGGBM.pptx

- Graphicalabstract.pptx 